\title{
Erratum: Hacking Alice's box in continuous-variable quantum key distribution [Phys. Rev. A 98, 062319 (2018)]
}

\author{
Jason Pereira $[$ and Stefano Pirandola
}

(Received 5 October 2020; published 28 December 2020)

DOI: 10.1103/PhysRevA.102.069903

In Sec. II B, the final paragraph, beginning "It is helpful to clarify...," has typographical errors in two of the inline equations. Specifically, $\beta=\eta(\alpha+n)$ should $\operatorname{read} \beta=\sqrt{\eta}(\alpha+n)$, and $\beta=\eta^{\prime}\left(k \alpha+n^{\prime}\right)$ should read $\beta=\sqrt{\eta^{\prime}}\left(k \alpha+n^{\prime}\right)$. These are typographical errors and do not affect any future calculations.

In Sec. III, the paragraph beginning "If the modulator can be surrounded..." has an incorrect proof of Eqs. (41) and (42). Specifically, the two sentences beginning with "The conditional state..." and including Eqs. (39) and (40) are wrong (up to the sentence beginning with "If the Trojan horse mode..."). They should instead read as follows:

The conditional state $\psi_{E_{S}, E_{I}} \mid \alpha$ received by Eve after the side channel (conditioned on Alice's value) is an attenuated two-mode squeezed vacuum (TMSV) state. It has the covariance matrix,

$$
V_{E_{S}, E_{I}} \mid \alpha=\left(\begin{array}{cc}
(2 \bar{n}+1) \mathbf{1} & 2 \sqrt{\bar{n}^{\prime}(\bar{n}+1)} \mathbb{Z} \\
2 \sqrt{\overline{n^{\prime}}(\bar{n}+1)} \mathbb{Z} & \left(2 \overline{n^{\prime}}+1\right) \mathbf{1}
\end{array}\right),
$$

where $\bar{n}$ is the photon number of the TMSV state prior to the side channel, $\bar{n}^{\prime}$ is some positive real number less than $\bar{n}, E_{S}$ is the signal mode, and $E_{I}$ is the idler mode.

Let $\psi_{E_{S} E_{I} P} \mid \alpha$ be the purification of $\psi_{E_{S}, E_{I}} \mid \alpha$. Eve does not hold the purifying mode $P$, but if she did, it could only help her. Therefore, let us assume she is given it; this is equivalent to saying she is given the other output of the beam splitter with its quadratures set to 0 . Then the modes $E_{I} P$ together purify $E_{S}$. Any one-mode thermal state can be purified by a TMSV [1]. Hence, there exists some unitary acting only on the purifying systems $E_{I}$ and $P$ that results in a TMSV on the modes $E_{S} E_{I}$ with $\bar{n}^{\prime}$ photons per mode (and a vacuum state on $P$ ).

All subsequent equations still hold.

The next paragraph, which begins "The expression for the maximum..." confuses upper and lower bounds and should instead read as follows:

The expression for the maximum secret key rate in this case is a lower bound: Giving Eve access to the purification mode $P$ cannot decrease the Holevo bound on her mutual information, but it is not immediately obvious whether it increases it. It is, therefore, not obvious whether this lower bound is tight, or whether the power of the side channel would be even further reduced by the attenuation; this is a question that is open to further study.

[1] C. Weedbrook, S. Pirandola, R. Garcia-Patron, N. J. Cerf, T. C. Ralph, J. H. Shapiro, and S. Lloyd, Rev. Mod. Phys. 84, 621 (2012). 